\title{
5FU resistance caused by reduced fluoro-deoxyuridine monophosphate and its reversal using deoxyuridine
}

\author{
RYUTARO MORI, MANABU FUTAMURA, TOSHIYUKI TANAHASHI, YOSHIHIRO TANAKA, \\ NOBUHISHA MATSUHASHI, KAZUYA YAMAGUCHI and KAZUHIRO YOSHIDA
}

Department of Surgical Oncology, Gifu University Graduate School of Medicine, Gifu 501-1194, Japan

Received January 25, 2017; Accepted May 11, 2017

DOI: $10.3892 / 01.2017 .6512$

\begin{abstract}
The mechanism of 5-fluorouracil (5FU) resistance was investigated, focusing on the level of thymidylate synthase (TS) ternary complex formed with fluoro-deoxyuridine monophosphate (FdUMP). MKN45 and 5FU-resistant MKN45/F2R cells were treated with 5FU and fluoro-deoxyuridine (FdU) in combination with deoxyuridine (dU) and thymidine (dT). Subsequently, the levels of ternary complex were determined by western blotting and the cell viability was calculated using an MTT assay. MKN45/F2R cells exhibited 5FU resistance (56.2-fold relative to MKN45 cells), and demonstrated decreased orotate phosphoribosyltransferase (OPRT) and increased TS levels, requiring a higher concentration of 5FU to induce ternary complex formation than MKN45 cells. Following transfection of small interfering RNA against OPRT, MKN45 exhibited increased resistance to 5FU and decreased ternary complex formation subsequent to treatment with $5 \mathrm{FU}$, indicating that decreased OPRT led to increased 5FU resistance. However, MKN45/F2R also exhibited resistance to FdU, which can be converted to FdUMP without OPRT, and there was decreased ternary complex formation after treatment with FdU, indicating that the 5FU-resistant cells had the ability to decrease intracellular FdUMP. The addition of dU and thymidine $\mathrm{dT}$ to $5 \mathrm{FU}$ promoted the formation of ternary complexes and reversed 5FU resistance in MKN45/F2R cells, although $\mathrm{dT}$ inhibited the efficacy of raltitrexed (another TS inhibitor). These results suggested that 5FU-resistant cells had the ability to reduce intracellular FdUMP irrespective of
\end{abstract}

Correspondence to: Dr Ryutaro Mori, Department of Surgical Oncology, Gifu University Graduate School of Medicine, 1-1 Yanagido, Gifu 501-1194, Japan

E-mail: moriry52@gmail.com

Abbreviations: 5FU, 5-fluorouracil; FdUMP, 5-fluorodeoxyuridine monophosphate; dU, deoxyuridine; dUMP, deoxyuridine monophosphate; dTMP, thymidine monophosphate; $\mathrm{dT}$, thymidine; TS, thymidylate synthase; OPRT, orotate phosphoribosyltransferase

Key words: stomach neoplasms, 5FU, drug resistance, fluorodeoxyuridylate decreased OPRT, which led to resistance to 5FU. This resistance was then inhibited by treatment with $\mathrm{dT}$ or $\mathrm{dU}$.

\section{Introduction}

Despite recent advances in the development of diagnostic tools for gastric cancer, many patients with gastric cancer continue to be diagnosed at a late stage, and even following curative surgery, recurrent tumors frequently occur. Therefore, gastric cancer remains a major cause for cancer-associated mortality worldwide (1) and the development of novel drug therapies for gastric cancer is important.

$5 \mathrm{FU}$ is currently a key drug for adjuvant therapy following curative surgery (2-4) and for the treatment of metastatic gastric cancer $(5,6)$. Three mechanisms have been proposed for its action: Incorporation into RNA (7), incorporation into DNA (8) and the inhibition of thymidine synthase (TS) leading to the inhibition of DNA de novo synthesis by forming a ternary complex composed of TS, 5,10-methylenetetrahydrofolate (CH2THF) and fluoro-deoxyuridine monophosphate (FdUMP) (9). The first step in the activation of 5FU is the phosphorylation of 5FU by orotate phosphoribosyltransferase (OPRT), which metabolizes 5FU to 5-fluorouridine monophosphate (FUMP). FUMP is then metabolized into 5-fluorouridine triphosphate (FUTP), which can be incorporated into RNA and into 5-flurodeoxyuridine triphosphate (FdUTP), which can be incorporated into DNA (10). Although the incorporation into RNA and DNA is certainly an important aspect of the mechanism of action of 5FU, the potential mechanism that has received the most focus is the inhibition of TS by the formation of ternary complexes, as a number of chemotherapeutic drugs similarly inhibit TS, including methotrexate (11), pemetrexate and raltitrexed (12). TS is the enzyme required to convert deoxyuridine monophosphate (dUMP) to thymidine monophosphate (dTMP); the inhibition of TS causes a lack of dTMP, leading to the inhibition of DNA de novo synthesis and cell death, a phenomenon termed 'thymineless death' (13).

Decreased levels of OPRT and increased levels of TS are thus considered major factors in the development of 5FU resistance (14). However, analysis of the expression of OPRT and TS in patients enrolled in the ACTS-GC trial, which investigated the efficacy of S-1 (5FU derivatives) for adjuvant therapy, revealed that increased TS was associated with a good 
prognosis and that the expression of OPRT was not associated with the prognosis at all (15).

These observations suggest that there may be unknown mechanisms of action involved in the development of 5FU resistance. Further investigation is therefore required to develop more effective drug therapies for gastric cancer. As $5 \mathrm{FU}$ is considered to be a TS inhibitor, 5FU resistance is associated with the decreased intracellular concentration of FdUMP (16). Therefore, in the present study, the mechanism of 5FU resistance was investigated, focusing on the amount of TS ternary complex formed by FdUMP.

\section{Materials and methods}

Drugs. 5FU was kindly provided by Kyowa Hakko Kirin Co., Ltd. (Tokyo, Japan). Raltitrexed, deoxyuridine (dU), and leucovorin were purchased from Sigma-Aldrich (Merck KGaA, Darmstadt, Germany). Thymidine (dT) was purchased from Wako Pure Chemical Industries, Ltd. (Osaka, Japan).

Cell lines and cell culture. MKN45 cells (a poorly differentiated gastric cancer cell line, kindly provided by Hiroshima Univiersity (Hiroshima, Japan), were cultured in RPMI-1640 with $10 \%$ fetal bovine serum (FBS; both from Wako Pure Chemical Industries, Ltd.) and sodium pyruvate (Sigma-Aldrich; Merck KGaA). MKN45/F2R cells are a 5FU-resistant cell line that was established by continuously exposing MKN45 cells to increasing concentrations $(0.1-2 \mu \mathrm{M})$ of 5FU over a year, as previously described (17). These cells were routinely maintained in RPMI-1640 with $10 \%$ FBS containing $2 \mu \mathrm{M} 5 \mathrm{FU}$, and prior to the study, the resistant cells were cultured in drug-free RPMI-1640 with $10 \%$ FBS for at least two weeks to eliminate the effects of 5FU in the experiments. The two cell lines were incubated in a humidified atmosphere of $5 \% \mathrm{CO}_{2}$ at $37^{\circ} \mathrm{C}$.

Western blot analysis and antibodies. The cells were lysed in radioimmunoprecipitation assay buffer (Sigma-Aldrich; Merck KGaA) for 15 min on ice. The protein concentration of the lysates was measured using a Bio-Rad Protein Assay Dye Reagent Concentrate (Bio-Rad Laboratories, Inc., Hercules, CA, USA). The cell lysates were boiled in sample buffer solution (Wako Pure Chemical Industries, Ltd.). Total cell protein extracts (10 $\mu \mathrm{g} /$ lane) were separated by $10 \%$ SDS-PAGE using SuperSep ${ }^{\mathrm{TM}}$ ACE (Wako Pure Chemical Industries, Ltd.) and electrophoretically transferred onto polyvinyl difluoride (PVDF) membranes. The membranes were blocked with PVDF blocking reagent (Toyobo Co., Ltd., Osaka, Japan) for $1 \mathrm{~h}$. The membranes were then incubated with primary antibodies, such as $\beta$-actin (13E5) rabbit mAb (cat. no. 4970; Cell Signaling Technology, Danvers, MA, USA; 1:5,000), anti-Thymidylate Synthase, clone TS106 (cat. no. MAB4130; EMD Millipore, Billerica, MA, USA; 1:5,000) or anti-OPRT antibody (kindly provided by Taiho Pharmaceutical Company, Tokyo, Japan; 1:10,000) overnight at $4^{\circ} \mathrm{C}$. The primary antibodies were diluted with Can Get Signal Solution 1 (Toyobo Co., Ltd.). The membranes were then washed with Dako Washing Buffer (Agilent Technologies, Inc., Santa Clara, CA, USA) and incubated with goat anti-Mouse $\operatorname{IgG}$, Peroxidase Conjugated, heavy chain + light chain (cat. no. AP124P; EMD
Millipore) or goat anti-rabbit IgG, Peroxidase Conjugate (cat. no. AP132P; EMD Millipore) diluted to 1:25,000 with Can Get Signal Solution 2 (Toyobo Co., Ltd.) for $1 \mathrm{~h}$ at room temperature. Immunoreactive proteins were visualized with the ImmunoStar LD reagent (Wako Pure Chemical Industries, Ltd.) and images were captured using a GeneGnome HR system (Syngene Europe, Cambridge, UK).

MTT assay for the effects of 5FU and raltitrexed. Cell growth was assessed by an MTT assay. A total of $5 \times 10^{3}$ cells were seeded into each well of 96-well plates and cultured for $24 \mathrm{~h}$ at $37^{\circ} \mathrm{C}$. The cells were treated with raltitrexed, dU or dT for $72 \mathrm{~h}$, after which the culture medium was removed and $100 \mu \mathrm{l}$ of a $0.5 \mathrm{mg} / \mathrm{ml}$ solution of MTT (Sigma-Aldrich; Merck $\mathrm{KGaA}$ ) was added to each well. The plates were then incubated for $4 \mathrm{~h}$ at $37^{\circ} \mathrm{C}$. The MTT solution was replaced with $100 \mu \mathrm{l}$ of dimethyl sulfoxide (Wako Pure Chemical Industries, Ltd.) per well and the absorbance at $540 \mathrm{~nm}$ was measured using an Envision 2104 Multilabel Reader (PerkinElmer, Inc., Waltham, MA, USA). Each assay was repeated three times.

Statistical analysis. The mean Half-maximal inhibitory concentration $\left(\mathrm{IC}_{50}\right)$ values were calculated based on each of the results of the MTT assays using the Microsoft Excel 2010 (Microsoft Corporation, Redmond, Washington, USA). Results are presented as the mean \pm standard error. The significance of differences in $\mathrm{IC}_{50}$ values were determined by Student's t-test using JMP 8 (SAS Institute Inc., Cary, NC, USA). P<0.05 was considered to indicate a statistically significant difference.

Transfection and small interfering RNA (siRNA) experiments for OPRT. MKN45 cells were cultured in RPMI-1640 with $10 \%$ FBS without antibiotics for $24 \mathrm{~h}$ to $50-70 \%$ confluence prior to transfection. Cells were then transfected with siRNA oligonucleotides using Lipofectamine RNAiMAX (Invitrogen; Thermo Fisher Scientific, Inc., Waltham, MA, USA) at a concentration of $40 \mathrm{nmol} / 1$ siRNA in serum-free Opti-MEM (Invitrogen; Thermo Fisher Scientific, Inc.) for 48 h. siRNA oligonucleotides for OPRT (Stealth RNAi; cat. no. 10620319-281527 A02 and 10620318-281527 A03) and negative control oligonucleotides (Stealth RNAi siRNA Negative Control; cat. no. 452002) were purchased from Invitrogen (Thermo Fisher Scientific, Inc.).

\section{Results}

Decreased OPRT caused decreased formation of ternary complexes, leading to $5 F U$ resistance. MKN45/F2R cells were previously established as 5FU-resistant cells with a demonstrated $\mathrm{IC}_{50}$ of $54.5 \pm 6.76 \mu \mathrm{M} 5 \mathrm{FU}$, representing a 56.2 -fold increase in resistance compared with parental MKN45 cells $\left(\mathrm{IC}_{50}, 0.97 \pm 0.17 \mu \mathrm{M} ; \mathrm{P}<0.05\right)(17)$. A western blot analysis demonstrated that the expression of TS was increased, whereas the level of OPRT was decreased in MKN45/F2R cells compared with MKN45 cells. Following treatment with $5 \mathrm{FU}(1,10$ or $100 \mu \mathrm{M})$ for $24 \mathrm{~h}$, the upper immunoreactive band of TS, which represents TS in ternary complexes and is correlated with the intracellular concentration of FdUMP (18), at $1 \mu \mathrm{M} 5 \mathrm{FU}$ was decreased in MKN45/F2R cells compared with MKN45 cells (Fig. 1A). 
A

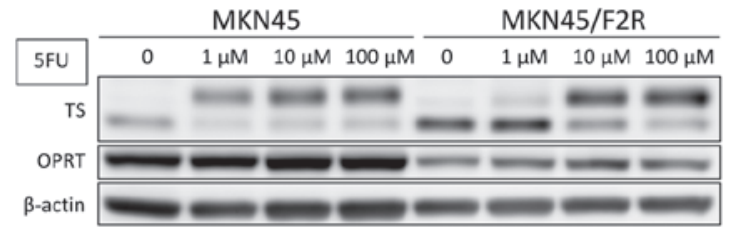

B

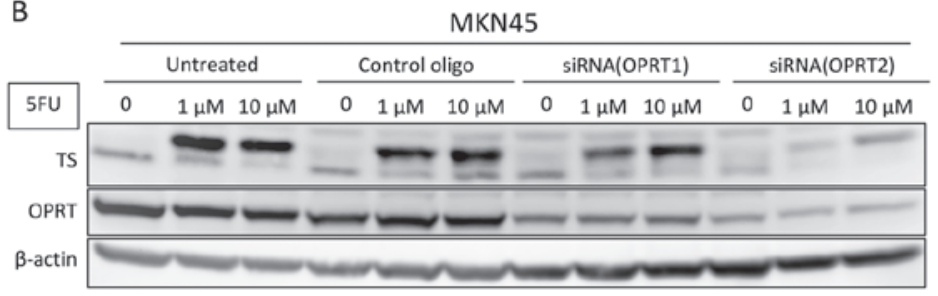

C

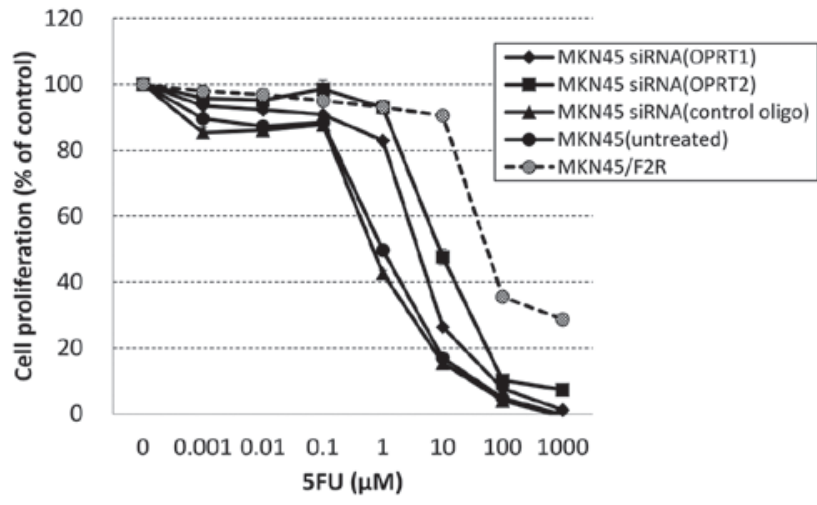

D
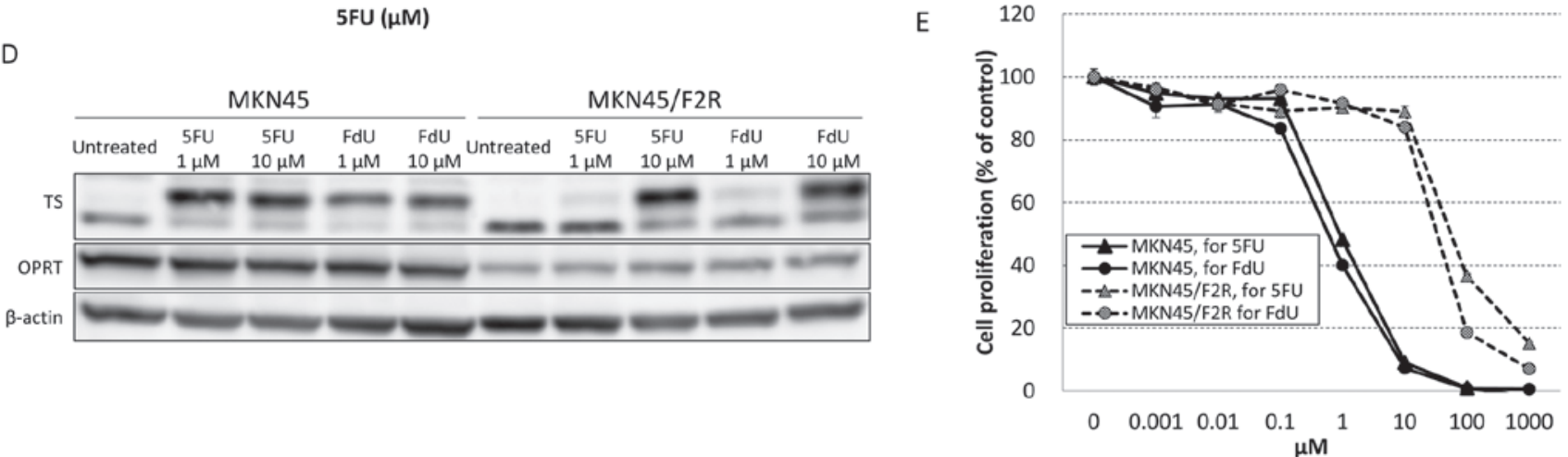

Figure 1. Effect of 5FU, FdU and OPRT siRNA on the expression of TS and OPRT, and the viability of MKN45 and MKN45/F2R cells. (A) Western blot analysis of TS and OPRT protein expression following treatment with 5FU in MKN45 and MKN45/F2R cells. (B) Western blot analysis of TS and OPRT protein expression following transfection with OPRT siRNA and treatment with 5FU in MKN45 cells. (C) MTT assay for the effect of 5FU treatment on the relative proliferation of MKN45 cells, MKN45 cells transfected with OPRT siRNA and MKN45/F2R cells. (D) Western blot analysis for TS and OPRT protein expression following treatment with 5FU and FdU in MKN45 and MKN45/F2R cells. (E) MTT assay for the effect of 5FU and FdU treatment on the relative proliferation of MKN45 and MKN45/F2R cells. 5FU, 5-fluorouracil; FdU, fluoro-deoxyuridine; OPRT, orotate phosphoribosyltransferase; siRNA, small interfering RNA; TS, thymidylate synthase.

Following the transfection of two types of siRNA against OPRT (OPRT 1 and 2) into MKN45 cells, a western blot analysis demonstrated that the greater the extent of OPRT knockdown, the greater the decrease in the upper band of TS at $1 \mu \mathrm{M} 5 \mathrm{FU}$ (Fig. 1B). The results of an MTT assay indicated an significantly increased $5 \mathrm{FU} \mathrm{IC} \mathrm{I}_{50}$ in MKN45 cells following siRNA transfection (OPRT1, 3.82 $\pm 0.22 \mu \mathrm{M} ; \mathrm{P}<0.05)$; OPRT2, $8.85 \pm 2.93 \mu \mathrm{M} ; \mathrm{P}<0.05$; Fig. 1C), which was consistent with the results of the western blot analysis. The results indicated that decreased expression of OPRT may have caused a decrease in the formation of ternary complexes, leading to $5 \mathrm{FU}$ resistance.

The formation of ternary complexes in 5FU-resistant cells decreased following treatment with 5FU or FdU. Fluoro-deoxyuridine (FdU) may be converted to FdUMP without OPRT and form ternary TS complexes (19). A western blot analysis revealed that the upper band of TS at $1 \mu \mathrm{M}$ of FdU in MKN45/F2R was decreased compared with MKN45 cells, similar to the findings for 5FU (Fig. 1D). An MTT assay demonstrated there was a 56.1-fold increase in resistance to
FdU $\left(\mathrm{IC}_{50}, 33.1 \pm 2.34 \mu \mathrm{M}\right)$ compared with MKN45 cells $\left(\mathrm{IC}_{50}\right.$, $0.59 \pm 0.10 \mu \mathrm{M})$, which was similar to the extent of $5 \mathrm{FU}$ resistance (Fig. 1E). These results indicated that the 5FU-resistant cells were able to reduce the levels of intracellular FdUMP irrespective of decreased OPRT levels.

$d U$ and $d T$ promoted the formation of ternary complexes and decreased $5 F U$ resistance in MKN45/F2R cells. High intracellular dUMP has been suggested to be the causative factor for $5 \mathrm{FU}$ resistance, as dUMP may competitively inhibit FdUMP (20). dTMP can also inhibit TS inhibitors, as TS is the enzyme that catalyzes the conversion of dUMP to dTMP. Therefore, $\mathrm{dU}$ and $\mathrm{dT}$ were used to increase the intracellular concentrations of dUMP and dTMP and investigate the influence of these molecules on 5FU.

A western blot analysis revealed that the combination of $1,000 \mu \mathrm{M}$ of dU with $1 \mu \mathrm{M}$ of $5 \mathrm{FU}$ or FdU increased the upper band of TS compared with 5FU or FdU alone (Fig. 2A), and similar results were observed when $1,000 \mu \mathrm{M}$ of dT was combined with 5FU or FdU (Fig. 2B). An MTT assay 
A

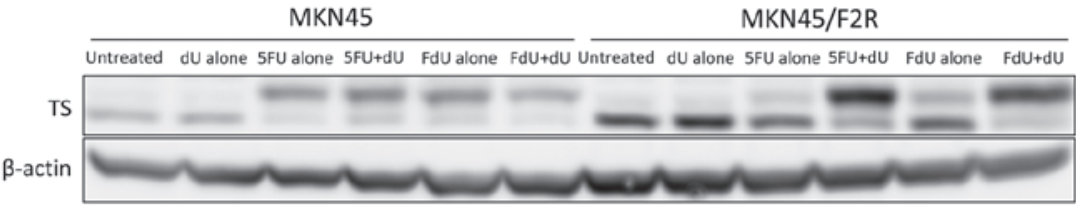

B

MKN45

MKN45/F2R

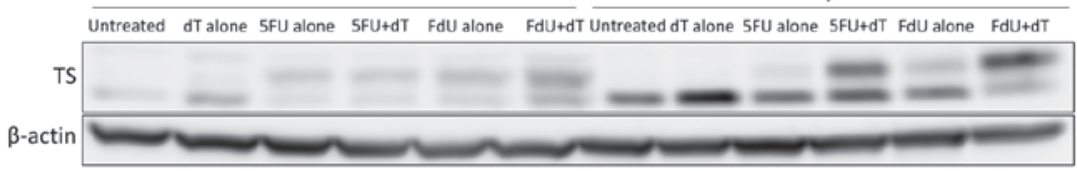

C

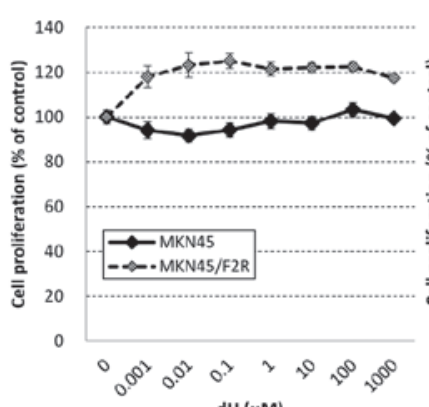

$d U(\mu M)$
D

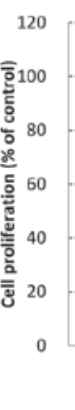

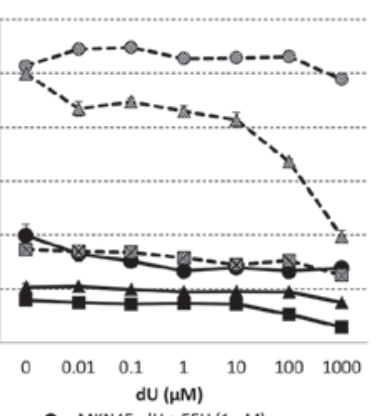

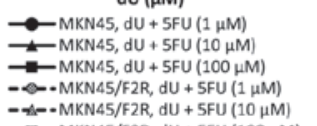

E

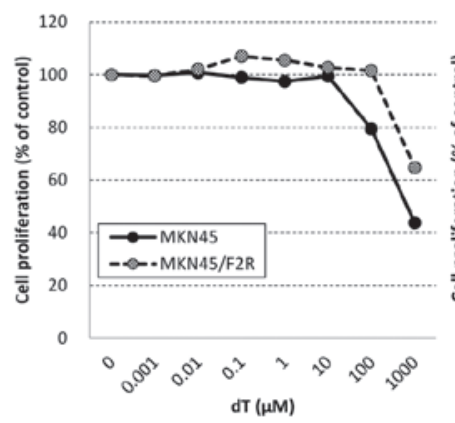

F

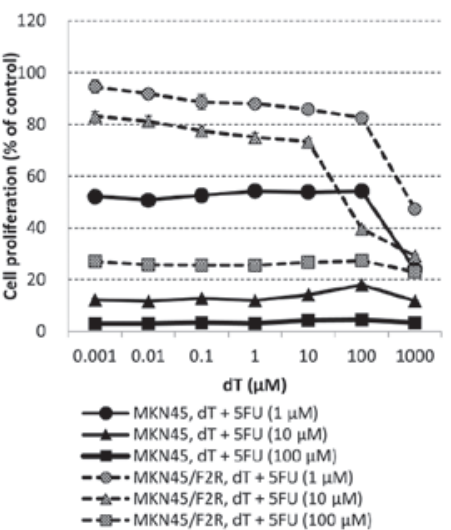

Figure 2. Combination of dT and dU with 5FU in MKN45 and MKN45/F2R cells. Western blot analysis for TS protein expression following treatment with (A) $5 \mathrm{FU}(1 \mu \mathrm{M})$ and $\mathrm{FdU}(1 \mu \mathrm{M})$ with/without dU $(1,000 \mu \mathrm{M})$ and (B) $5 \mathrm{FU}(1 \mu \mathrm{M})$ and $\mathrm{FdU}(1 \mu \mathrm{M})$ with/without dT $(1,000 \mu \mathrm{M})$. MTT assay for the relative proliferation of MKN45 and MKN45/F2R cells treated with (C) dU alone, (D) dU with 5FU, (E) dT alone and (F) dT with 5FU. dT, thymidine; dU, deoxyuridine; 5FU, 5 -fluorouracil; TS, thymidylate synthase; FdU, fluoro-deoxyuridine.

demonstrated that $\mathrm{dU}$ alone did not have a cytotoxic effect on MKN45 or MKN45/F2R cells (Fig. 2C), and the addition of $\mathrm{dU}$ did not inhibit the cytotoxicity of $5 \mathrm{FU}$; the addition of $>1 \mu \mathrm{M}$ of dU actually decreased the $5 \mathrm{FU}$ resistance at $10 \mu \mathrm{M}$ of 5FU in MKN45/F2R cells (Fig. 2D). The combination of dU with 5FU exhibited no effect in MKN45 cells (Fig. 2D). These results were consistent with the results of the western blot analysis. Furthermore, dT alone exerted a cytotoxic effect on MKN45 and MKN45/F2R cells IC I0 MKN45 cells, $671 \mu \mathrm{M}$; $\mathrm{IC}_{50}$ in MKN45/F2R cells, $>1,000 \mu \mathrm{M}$; Fig. 2E). The addition of dT also did not inhibit the cytotoxicity of $5 \mathrm{FU}$, instead slightly decreasing the $5 \mathrm{FU}$ resistance at $10 \mu \mathrm{M}$ of $5 \mathrm{FU}$ in MKN45/F2R cells (Fig. 2F). These results suggested that dU and dT did not inhibit the cytotoxicity of 5FU, and may have reversed 5FU resistance in 5FU-resistant cells.

Thymidine decreased the cytotoxicity of TS inhibitors. The cytotoxicity of TS inhibitors can be inhibited by dT, as dT can be converted to dTMP without TS, a process known as 'thymidine rescue' (21). As aforementioned, 5FU was not inhibited by dT, although $5 \mathrm{FU}$ is considered as a TS inhibitor. Therefore, dT treatment was combined with raltitrexed, a specific TS inhibitor, to confirm if dT may have inhibited TS inhibitors.

MKN45/F2R cells exhibited raltitrexed resistance and the $\mathrm{IC}_{50} \mathrm{~s}$ for raltitrexed alone in MKN45 and MKN45/F2R cells were 1.12 and $5.13 \mathrm{nM}$, respectively (Fig. 3A). The addition of dU to raltitrexed had no effect on MKN45 or MKN45/F2R cells (Fig. 3B), and the addition of dT inhibited the cytotoxicity of raltitrexed at concentrations exceeding $0.1 \mu \mathrm{M}$ in MKN45 cells and exceeding $10 \mu \mathrm{M}$ in MKN45/F2R cells (Fig. 3C). These results indicated that dT exhibited the ability to inhibit TS inhibitors, and the cytotoxic effect of $5 \mathrm{FU}$ is not mediated by 'thymineless death'.

Leucovorin enhanced the cytotoxic effect of $5 F U$ in parental MKN45 cells. Leucovorin is easily metabolized to CH2THF, a component of ternary complexes, and has been used to enhance the efficacy of 5FU against colorectal cancer (22). To confirm the difference in the mechanism between leucovorin and $\mathrm{dU}$, leucovorin was combined with $5 \mathrm{FU}$.

A western blot analysis demonstrated that the addition of leucovorin to $5 \mathrm{FU}$ increased the upper band of TS in parental MKN45 cells. However, it had no effect on MKN45/F2R cells (Fig. 4A). An MTT assay demonstrated that leucovorin alone did not have a cytotoxic effect on MKN45 or MKN45/F2R cells (Fig. 4B), and whereas combination of $>0.01 \mu \mathrm{M}$ of leucovorin increased the cytotoxic effect of 5FU in parental MKN45 cells, there was no effect on MKN45/F2R cells (Fig. 4C), consistent with the results of the western blot analysis. These results indicated that the mechanism for the reversal of $5 \mathrm{FU}$ resistance by $\mathrm{dU}$ and $\mathrm{dT}$ differed from the mechanism for enhancing the efficacy of $5 \mathrm{FU}$ by leucovorin. Furthermore, the results confirmed that the cause of the decreased amount of ternary complex in 5FU-resistant cells was unlikely to be a lack of $\mathrm{CH} 2 \mathrm{THF}$. 
A
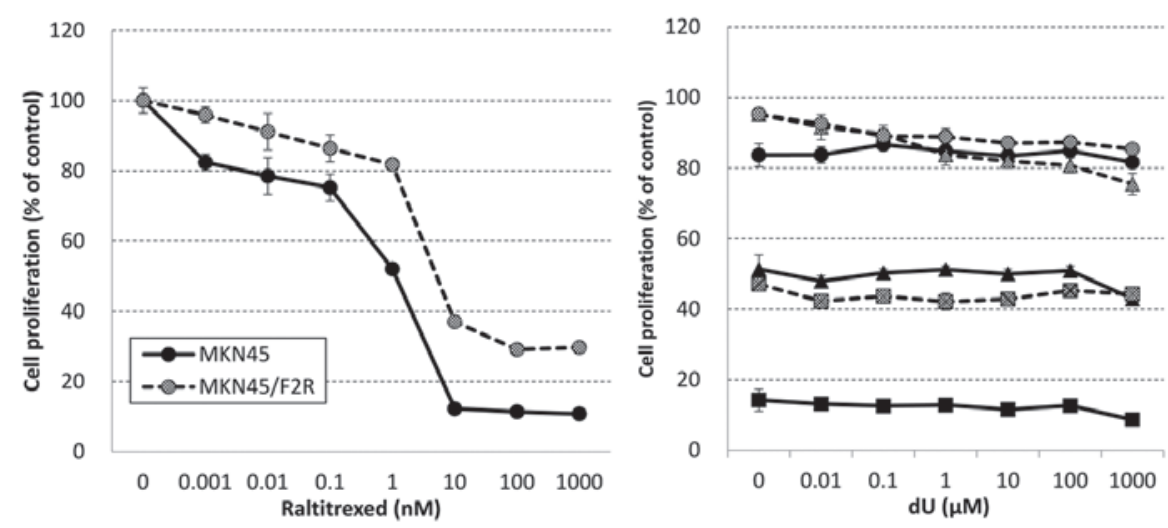

$\longrightarrow$ MKN45, dU + raltitrexed $(0.1 \mathrm{nM})$ -MKN45, dU + raltitrexed (1 $\mathrm{nM})$ $\rightarrow-M K N 45, d U+$ raltitrexed $(10 \mathrm{nM})$ - - -MKN45/F2R, dU + raltitrexed $(0.1 \mathrm{nM})$ - - MKN45/F2R, dU + raltitrexed (1 nM)

- $\$$-MKN45/F2R, dU + raltitrexed (10 nM)
C

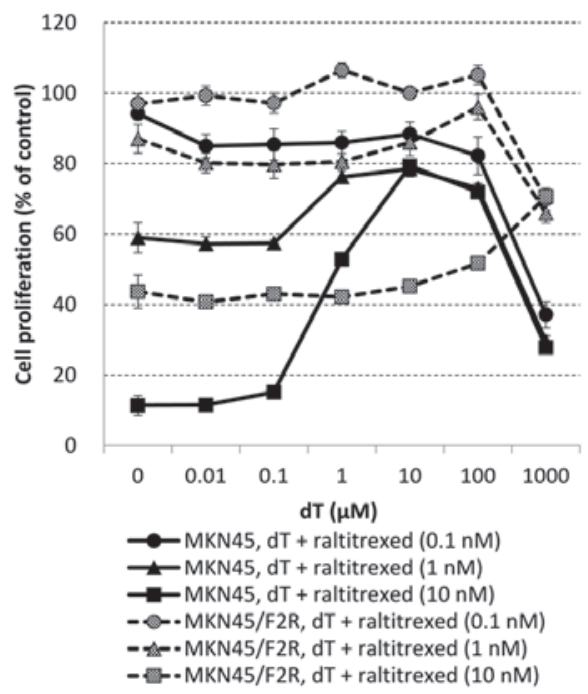

Figure 3. Resistance to raltitrexed in 5-fluorouracil-resistant MKN45/F2R cells and the inhibition of raltitrexed by dT. MTT assay for the relative proliferation of MKN45 and MKN45/F2R cells treated with (A) raltitrexed, (B) dU with raltitrexed, and (C) dT. dT, thymidine; dU, deoxyuridine.

A MKN45 MKN45/F2R

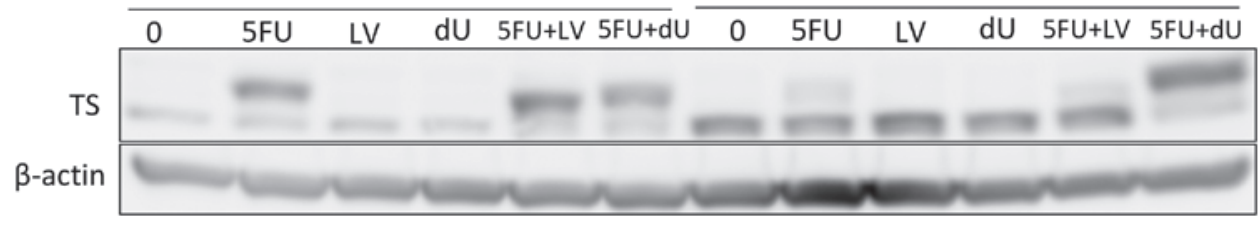

B

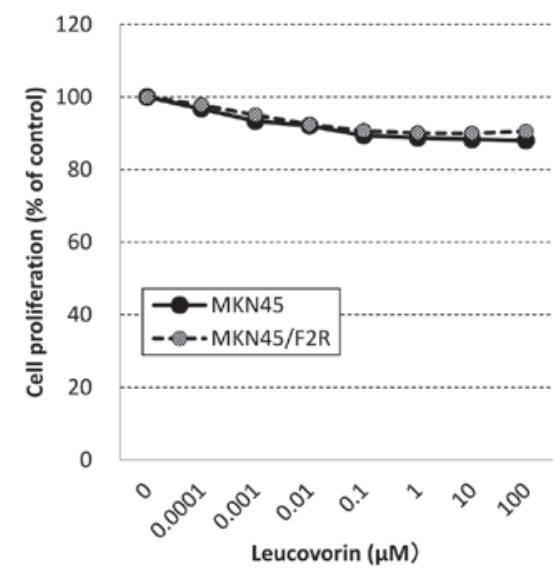

C

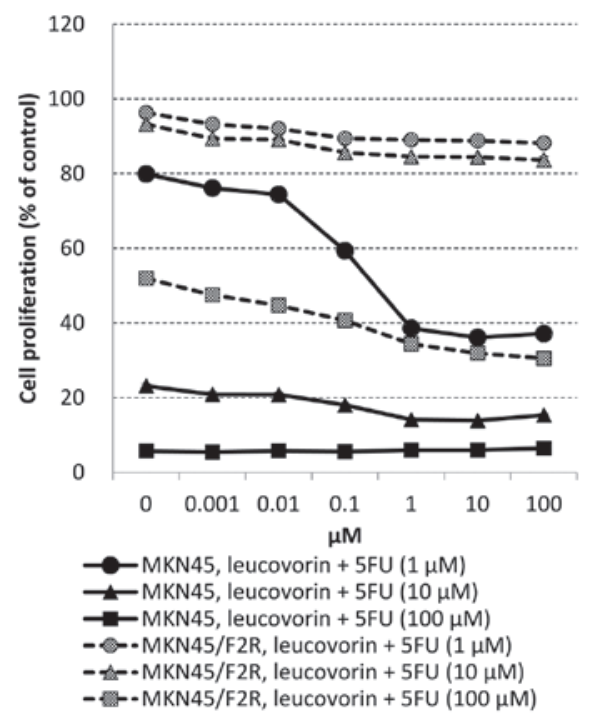

Figure 4. Enhancement of the efficacy of 5FU by LV. (A) Western blot analysis for TS protein expression following treatment with $5 \mathrm{FU}, \mathrm{LV}$, their combination or 5FU with dU in MKN45 and MKN45/F2R cells. MTT assay for the relative proliferation of MKN45 and MKN/F2R cells treated with (B) LV and (C) LV with 5FU in MKN45 and MKN45/F2R cells. 5FU, 5-fluorouracil; LV, leucovorin; TS, thymidylate synthase; dU, deoxyuridine.

\section{Discussion}

In the present study, the mechanism of 5FU resistance was investigated with a focus on the amount of TS ternary complex, which correlates with the intracellular concentration of FdUMP (16). It was identified that 5FU-resistant cells may acquire the ability to reduce intracellular FdUMP irrespective of decreased OPRT levels.

A lack of dTTP causes 'thymineless death' (13); as $5 \mathrm{FU}$ inhibits TS by forming ternary complexes, it has been hypothesized that the major mechanism of action for $5 \mathrm{FU}$ is thymineless death caused by TS inhibition (10). However, 
the data of the present study suggest that cell death caused by $5 \mathrm{FU}$ was not induced by thymineless death alone, as $5 \mathrm{FU}$ cytotoxicity could not be inhibited by dT administration; dT actually somewhat decreased the 5FU resistance of 5FU-resistant cells.

OPRT has been reported as an important factor for $5 \mathrm{FU}$ resistance and there are a number of reports stating that decreased OPRT levels cause 5FU resistance $(17,23,24)$, which does not contradict the results of the present study. OPRT is required for the first step of 5FU activation; decreased OPRT levels lead to decreased intracellular FdUMP. Therefore, decreased OPRT levels lead to the reduced inhibition of TS. However, in the present study, 5FU-resistant cells demonstrated decreased TS ternary complex formation following treatment not only with 5FU, but also with FdU, which can be converted to FdUMP without OPRT. Therefore, the resistant cells may have the ability to reduce intracellular FdUMP irrespective of decreased OPRT levels.

In the present study, dT slightly decreased $5 \mathrm{FU}$ resistance whereas dU greatly decreased 5FU resistance. There have been previous reports describing the enhanced cytotoxicity of $5 \mathrm{FU}$ due to deoxyribonucleosides, including dT and dU $(25,26)$. In these reports, the mechanism of this enhancement was speculated to be the inhibition of ribonucleoside reductase (RR) by a high concentration of deoxyribonucleosides, leading to an increase in the incorporation of 5FU into RNA. However, the data of the present study revealed that 5FU-resistant cells exhibited resistance to FdU, which is not easily incorporated into RNA, similar to the extent of 5FU resistance, suggesting that the incorporation into RNA is not of high importance in 5FU resistance. Furthermore, dT and dU appeared to inhibit the decrease in intracellular FdUMP in 5FU-resistant cells, as the addition of $\mathrm{dT}$ and $\mathrm{dU}$ increased the ternary complex formation following treatment with $5 \mathrm{FU}$ or FdU.

To our knowledge, there have been no previous reports describing a direct association between the ability of cells to decrease intracellular FdUMP concentration and 5FU resistance. We speculate that a potential mechanism for reducing FdUMP may be dephosphorylation by 5'-nucleotidases and phosphatases. The dephosphorylation of nucleosides, an important component of nucleoside analog metabolism, and the overexpression of nucleotidases is associated with an increased $\mathrm{IC}_{50}$ for the nucleoside analogs cytosine arabinoside and chloro-deoxyadenosin (27). The overexpression of nucleotidase may also be associated with an increased $\mathrm{IC}_{50}$ for 5FU (27), but the association of the dephosphorylation of FdUMP with 5FU resistance has not been clarified. The present study hypothesized that a large amount of deoxyribonucleosides, such as dU and dT, may inhibit the activity of nucleotidases and phosphatases; this mechanism may be involved in the reversal of 5FU resistance.

In conclusion, it was identified in the present study that a reduction in the intracellular FdUMP level is likely to be a major mechanism involved in the resistance to 5FU, and this reduction could be inhibited by $\mathrm{dT}$ or $\mathrm{dU}$ treatment. Further investigations, regarding the mechanism underlying the decrease in intracellular FdUMP and the contribution of nucleotidase to 5FU resistance, are required. The significance of these mechanisms also requires confirmation in a clinical setting.

\section{Acknowledgements}

This study was supported by Grants-in-Aid for Scientific Research (C) from the Ministry of Education, Culture, Sports, Science and Technology (grant no. 26461973), and Taiho Pharmaceutical Co., Ltd. The authors wish to disclose the following potential conflicts of interest: Dr Kazuhiro Yoshida has received grants and personal fees from Taiho Pharmaceutical Co., Ltd., Pfizer Inc., Chugai Pharmaceutical Co., Ltd., and Yakult Honsha Co., Ltd.; grants from Bristol-Myers Squibb and Kyowa Hakko Kirin Co., Ltd.; honoraria from Pfizer Inc., Chugai Pharmaceutical Co., Ltd., Kyowa Hakko Kirin Co., Ltd., Taiho Pharmaceutical Co., Ltd. and Yakult Honsha Co., Ltd., and has had a consultant or advisory relationship with La Roche, Ltd. and Taiho Pharmaceutical Co., Ltd.

\section{References}

1. Torre LA, Bray F, Siegel RL, Ferlay J, Lortet-Tieulent J and Jemal A: Global cancer statistics, 2012. CA Cancer J Clin 65: 87-108, 2015

2. Sakuramoto S, Sasako M, Yamaguchi T, Kinoshita T, Fujii M, Nashimoto A, Furukawa H, Nakajima T, Ohashi Y, Imamura H, et al: Adjuvant chemotherapy for gastric cancer with S-1, an oral fluoropyrimidine. N Engl J Med 357: 1810-1820, 2007.

3. Sasako M, Sakuramoto S, Katai H, Kinoshita T, Furukawa H, Yamaguchi T, Nashimoto A, Fujii M, Nakajima T and Ohashi Y: Five-year outcomes of a randomized phase III trial comparing adjuvant chemotherapy with $\mathrm{S}-1$ versus surgery alone in stage II or III gastric cancer. J Clin Oncol 29: 4387-4393, 2011.

4. Tsuburaya A, Yoshida K, Kobayashi M, Yoshino S, Takahashi M, Takiguchi N, Tanabe K, Takahashi N, Imamura H, Tatsumoto N, et al: Sequential paclitaxel followed by tegafur and uracil (UFT) or S-1 versus UFT or S-1 monotherapy as adjuvant chemotherapy for $\mathrm{T} 4 \mathrm{a} / \mathrm{b}$ gastric cancer (SAMIT): A phase 3 factorial randomised controlled trial. Lancet Oncol 15: 886-893, 2014.

5. Koizumi W, Narahara H, Hara T, Takagane A, Akiya T, Takagi M, Miyashita K, Nishizaki T, Kobayashi O, Takiyama W, et al: S-1 plus cisplatin versus $\mathrm{S}-1$ alone for first-line treatment of advanced gastric cancer (SPIRITS trial): A phase III trial. Lancet Oncol 9: 215-221, 2008.

6. Koizumi W, Kim YH, Fujii M, Kim HK, Imamura H, Lee KH, Hara T, Chung HC, Satoh T, Cho JY, et al: Addition of docetaxel to S-1 without platinum prolongs survival of patients with advanced gastric cancer: A randomized study (START). J Cancer Res Clin Oncol 140: 319-328, 2014.

7. Kufe DW and Major PP: 5-Fluorouracil incorporation into human breast carcinoma RNA correlates with cytotoxicity. J Biol Chem 256: 9802-9805, 1981.

8. Saito K, Nagashima H, Noguchi K, Yoshisue K, Yokogawa T, Matsushima E, Tahara T and Takagi S: First-in-human, phase I dose-escalation study of single and multiple doses of a first-in-class enhancer of fluoropyrimidines, a dUTPase inhibitor (TAS-114) in healthy male volunteers. Cancer Chemother Pharmacol 73: 577-583, 2014.

9. Park JG, Collins JM, Gazdar AF, Allegra CJ, Steinberg SM, Greene RF and Kramer BS: Enhancement of fluorinated pyrimidine-induced cytotoxicity by leucovorin in human colorectal carcinoma cell lines. J Natl Cancer Inst 80: 1560-1564, 1988.

10. Longley DB, Harkin DP and Johnston PG: 5-fluorouracil: Mechanisms of action and clinical strategies. Nat Rev Cancer 3: 330-338, 2003.

11. Goodsell DS: The molecular perspective: Methotrexate. Oncologist 4: 340-341, 1999.

12. Rose MG, Farrell MP and Schmitz JC: Thymidylate synthase: A critical target for cancer chemotherapy. Clin Colorectal Cancer 1: 220-229, 2002

13. Ahmad SI, Kirk SH and Eisenstark A: Thymine metabolism and thymineless death in prokaryotes and eukaryotes. Annu Rev Microbiol 52: 591-625, 1998.

14. Peters GJ, Laurensse E, Leyva A, Lankelma J and Pinedo HM: Sensitivity of human, murine and rat cells to 5-fluorouracil and 5'-deoxy-5-fluorouridine in relation to drug-metabolizing enzymes. Cancer Res 46: 20-28, 1986. 
15. Sasako M, Terashima M, Ichikawa W, Ochiai A, Kitada K, Kurahashi I, Sakuramoto S, Katai H, Sano T and Imamura H: Impact of the expression of thymidylate synthase and dihydropyrimidine dehydrogenase genes on survival in stage II/III gastric cancer. Gastric Cancer 18: 538-548, 2015.

16. Patel K, Yerram SR, Azad NA and Kern SE: A thymidylate synthase ternary complex-specific antibody, FTS, permits functional monitoring of fluoropyrimidines dosing. Oncotarget 3 : 678-685, 2012.

17. Tsutani Y, Yoshida K, Sanada Y, Wada Y, Konishi K, Fukushima M and Okada M: Decreased orotate phosphoribosyltransferase activity produces 5-fluorouracil resistance in a human gastric cancer cell line. Oncol Rep 20: 1545-1551, 2008.

18. Drake JC, Allegra CJ and Johnston PG: Immunological quantitation of thymidylate synthase-FdUMP-5,10-methylenetetrahydrofolate ternary complex with the monoclonal antibody TS 106. Anticancer Drugs 4: 431-435, 1993.

19. Levin RD and Gordon JH: Fluorodeoxyuridine with continuous leucovorin infusion. A phase II clinical trial in patients with metastatic colorectal cancer. Cancer 72: 2895-2901, 1993.

20. Myers CE, Young RC and Chabner BA: Biochemical determinants of 5-fluorouracil response in vivo. The role of deoxyuridylate pool expansion. J Clin Invest 56: 1231-1238, 1975.

21. Howell SB, Ensminger WD, Krishan A and Frei E III: Thymidine rescue of high-dose methotrexate in humans. Cancer Res 38 : 325-330, 1978
22. Francini G, Petrioli R, Lorenzini L, Mancini S, Armenio S, Tanzini G, Marsili S, Aquino A, Marzocca G, Civitelli S, et al: Folinic acid and 5-fluorouracil as adjuvant chemotherapy in colon cancer. Gastroenterology 106: 899-906, 1994.

23. Kodera Y, Ito S, Fujiwara M, Mochizuki Y, Nakayama G, Ohashi N, Koike M, Yamamura Y and Nakao A: Gene expression of 5-fluorouracil metabolic enzymes in primary gastric cancer: Correlation with drug sensitivity against 5 -fluorouracil. Cancer Lett 252: 307-313, 2007.

24. Isshi K, Sakuyama T, Gen T, Nakamura Y, Kuroda T, Katuyama T and Maekawa Y: Predicting 5-FU sensitivity using human colorectal cancer specimens: Comparison of tumor dihydropyrimidine dehydrogenase and orotate phosphoribosyl transferase activities with in vitro chemosensitivity to 5-FU. Int J Clin Oncol 7: 335-342, 2002.

25. Santelli G and Valeriote F: In vivo potentiation of 5-fluorouracil cytotoxicity against AKR leukemia by purines, pyrimidines, and their nucleosides and deoxynucleosides. J Natl Cancer Inst 64: 69-72, 1980.

26. Spiegelman S, Sawyer R, Nayak R, Ritzi E, Stolfi R and Martin D: Improving the anti-tumor activity of 5-fluorouracil by increasing its incorporation into RNA via metabolic modulation. Proc Natl Acad Sci USA 77: 4966-4970, 1980

27. Hunsucker SA, Spychala J and Mitchell BS: Human cytosolic 5'-nucleotidase I: Characterization and role in nucleoside analog resistance. J Biol Chem 276: 10498-10504, 2001. 